\title{
CONTENIDO DE MINERALES EN SEMILLA DE POBLACIONES NATIVAS DE FRIJOL COMÚN (Phaseolus vulgaris L.)
}

\author{
MINERAL CONTENT IN SEEDS OF NATIVE POPULATIONS \\ OF COMMON BEAN (Phaseolus vulgaris L.)
}

\begin{abstract}
Nancy Espinoza-García’, Raquel Martínez-Martínez', José L. Chávez-Servia ${ }^{2}$, Araceli M. Vera-Guzmán ${ }^{2}$, José C. Carrillo-Rodríguez ${ }^{1}$, Elena Heredia-García ${ }^{3}$ y Vicente A. Velasco-Velasco ${ }^{1}$
\end{abstract}

\begin{abstract}
${ }^{1}$ Instituto Tecnológico del Valle de Oaxaca. Ex-Hacienda Nazareno. 71230, Santa Cruz Xoxocotlán, Oaxaca, México. ${ }^{2}$ CIIDIR Unidad Oaxaca, Instituto Politécnico Nacional, Hornos \# 1003. 71230, Santa Cruz Xoxocotlán, Oaxaca, México. ${ }^{3}$ Campo Experimental El Bajío, Instituto Nacional de Investigaciones Forestales, Agrícolas y Pecuarias. Km 6.5 Carr. Celaya-San Miguel de Allende s/n. 38010, Celaya, Guanajuato, México.
\end{abstract}

*Autor de correspondencia (jchavezs@ipn.mx)

\section{RESUMEN}

El frijol común (Phaseolus vulgaris L.) es uno de los productos básicos en la dieta y gastronomía mexicana, no solo por la aportación de proteínas, carbohidratos, vitaminas, fibra y metabolitos secundarios, sino también por una amplia gama de macro y microelementos minerales como Fe y Zn, indispensables para la salud. El objetivo del estudio fue evaluar el contenido de minerales en semilla de una colección de 67 poblaciones nativas de frijol originarias de cuatro regiones de Oaxaca: Valles Centrales, Sierra Norte, Sierra Sur y Mixteca. Las poblaciones se sembraron en San Agustín Amatengo, Oaxaca, en primavera-verano 2014 bajo condiciones de temporal (secano), en un diseño de bloques completos al azar con cuatro repeticiones; a la cosecha se obtuvo una muestra compuesta de $500 \mathrm{~g}$ de semilla por accesión. La evaluación del contenido de minerales se hizo mediante espectrofotometría de absorción atómica y ultravioleta visible, con base en estándares de referencia. El análisis de varianza detectó diferencias significativas $(P \leq 0.01)$ entre y dentro de grupos, en todos los minerales evaluados. Las poblaciones de Valles Centrales y de la Sierra Norte destacaron por la frecuencia de valores altos en macro y micronutrientes. Las evaluaciones de macro y micronutrientes permitieron detectar altos valores en las poblaciones $\mathrm{P}-06, \mathrm{P}-60, \mathrm{P}-67, \mathrm{P}-73$, $\mathrm{P}-75$ y $\mathrm{P}-79$. En contenidos de Fe y Zn, cuyas deficiencias están asociadas con mala nutrición y problemas de salud, las poblaciones sobresalientes fueron P-01, P-03, P-04, P-06 y P-07.

Palabras clave: Acervos genéticos, espectrofotometría de absorción atómica, variación fenotípica.

\section{SUMMARY}

Common bean (Phaseolus vulgaris L.) is a basic staple product for the Mexican diet and gastronomy. It supplies proteins, carbohydrates, vitamins, fiber and secondary metabolites, as well as a wide range of mineral macro and microelements, such as Iron ( $\mathrm{Fe}$ ) and Zinc (Zn). Deficiencies of either element are associated to poor nutrition and health problems. This study evaluated mineral content in seeds from a collection of 57 common bean populations native from four regions of Oaxaca: Valles Centrales, Sierra Norte, Sierra Sur and Mixteca. The populations were sown in San Agustin Amatengo, Oaxaca during the 2014 Spring-Summer season under rainfed conditions using a randomized complete blocks design with four replications. At harvest time, a composite sample of $500 \mathrm{~g}$ of seed was obtained per population. The evaluation of mineral content was performed by atomic absorption and UVVis spectrophotometry based on reference standards. Analysis of variance detected significant differences $(P \leq 0.01)$ both among and within groups for all the evaluated minerals. The accession groups from Valles Centrales and Sierra Norte showed the highest frequency of large values in micro and macroelements. The evaluation also detected high values of macro and micronutrients in accessions P-06, P-60, P-67, P-73, P-75 and P-79. Regarding $\mathrm{Fe}$ and $\mathrm{Zn}$ content, the outstanding accessions were P-01, P-03, P-04, P-06 and P-07.

Index words: Genepools, atomic absorption spectrophotometry, phenotypic variation.

\section{INTRODUCCIÓN}

El frijol común (Phaseolus vulgaris L.) es una de las leguminosas de grano más importantes para consumo humano en el mundo. México ocupa el quinto lugar en la producción mundial con 1,273,957 t y tercero en América después de Brasil $(3,294,586$ t) y Estados Unidos de América (1,324,766 t) (FAOSTAT, 2015). En Brasil, Tanzania, Guatemala, Corea del Norte y Kenia, el consumo de frijol es algo mayor que en México (más de $10.5 \mathrm{~kg}$ anuales por persona), donde se estima un consumo promedio nacional de $10.38 \mathrm{~kg}$ por persona por año y una ingesta de $5.43 \mathrm{~g}$ de proteína por persona por día (SIAP, 2014). Además, 86.4 $\%$ de la producción nacional proviene de siembras de temporal (secano).

México es centro de origen y diversificación de P. vulgaris (Bitocchi et al., 2012; Singh et al., 1991), y la actual variabilidad genética y fenotípica de la forma domesticada en características de plantas, fisiológicos, de semilla y composición química, tiene como base la diversidad genética generada y preservada por las culturas precolombinas y campesinos actuales (Hernández-López et al., 2013; Lépiz et al., 2010). La variación de formas, tamaños y colores de semilla presentes en las diversas regiones donde se cultiva el frijol, es una muestra de la diversidad genética del frijol común cultivado en campos de los agricultores. Usualmente la caracterización de las poblaciones 
de frijol se efectúa mediante rasgos agromorfológicos (Vargas-Vázquez et al., 2008), marcadores moleculares (Vidal-Barahona et al., 2006), contenido de proteínas (Muñoz-Velázquez et al., 2009), antocianinas (Salinas-Moreno et al., 2005) y polifenoles (Espinosa-Alonso et al., 2006), entre otros aspectos.

El frijol aporta una fuente importante de proteínas, carbohidratos, vitaminas, fibra, metabolitos secundarios con alta actividad antioxidante y minerales (Guzmán et al., 2002). En trabajos previos, el contenido de minerales en frijol ha variado en función del material genético, manejo del cultivo y condiciones de almacenamiento (Beebe et al., 2000; Gouveia et al., 2014; Moraghan y Grafton, 2001). Raya-Pérez et al. (2014) determinaron diferencias significativas en contenido de minerales y proteína, entre dos variedades locales mexicanas de frijol, y Moraghan y Grafton (2001) encontraron en Dakota de Norte, EE. UU., efectos significativos de la interacción de ocho variedades y cinco sitios de evaluación en el contenido de minerales. En prácticas agrícolas, Astudillo y Blair (2008) determinaron que la concentración de hierro se incrementa con aplicaciones de fósforo mediante fertilizaciones, pero en zinc no hubo respuestas significativas con respecto a los testigos.

La ingesta de macro y microelementos minerales está asociada con la prevención de cáncer de próstata (Bidoli et al., 2005), reduce el riesgo de enfermedades coronarias (Bazzano et al., 2001), previene ataques al miocardio (Kabagambe et al., 2005); experimentalmente se encontraron efectos benéficos contra cáncer de colon en ratas (Reynoso et al., 2007) y las deficiencias de Fe y Zn son comúnmente asociadas con malnutrición de niños y mujeres embarazadas (Welch, 2008). En este contexto, el objetivo fue evaluar el contenido de minerales en semilla de una colección de poblaciones nativas de frijol (P. vulgaris L.) originarias de cuatro regiones de Oaxaca, México.

\section{MATERIALES Y MÉTODOS}

\section{Colecta y siembra de germoplasma}

Entre diciembre de 2013 y abril de 2014 se hizo una colecta de germoplasma de frijol en las regiones de Valles Centrales, Sierra Norte, Sierra Sur y Mixteca, del estado de Oaxaca; se obtuvieron 67 poblaciones nativas provenientes de 49 comunidades de 35 municipios indígenas de los grupos socioculturales Zapoteco, Mixe, Mixteco, Triqui y Chinanteco. Las regiones de colecta se localizan entre los $16^{\circ} 16^{\prime} 53^{\prime \prime}$ y $17^{\circ} 59^{\prime} 13^{\prime \prime} \mathrm{N}$, y los $95^{\circ} 56^{\prime} 44^{\prime \prime}$ y $98^{\circ} 03^{\prime} 08^{\prime \prime} \mathrm{O}$, en altitudes que van desde 133 hasta 2,730 msnm.

La ficha de colecta contiene el nombre del agricultor, georreferenciación del sitio de origen de la muestra, característica principal de la población, nombre local e información sobre el manejo agronómico. En el ciclo primavera-verano de 2014 se realizó la siembra y cultivo en asociación con maíz, en terrenos de un agricultor de San Agustín Amatengo, Oaxaca ( $16^{\circ} 31^{\prime} \mathrm{N}, 96^{\circ} 47^{\prime}$ O y 1360 msnm), bajo un diseño de bloques completos al azar con cuatro repeticiones y en condiciones de temporal (secano). El cultivo se condujo en forma tradicional (manual y yunta), se aplicó la fórmula de fertilización 60N-60P-60K y a la cosecha se obtuvo una muestra compuesta de $500 \mathrm{~g}$ de semilla por población, la cual se almacenó en refrigeración hasta su análisis de laboratorio.

\section{Evaluación del contenido de minerales en semilla}

A partir de las muestras de semilla se obtuvieron cenizas de cada muestra de acuerdo con el método 975.03B(a) de la AOAC (1990). Las cenizas se solubilizaron en un medio ácido para la extracción de los minerales $\mathrm{Cu}, \mathrm{Fe}, \mathrm{Mg}, \mathrm{Zn}$, $\mathrm{Na}, \mathrm{K}$, y Ca (mg/100 g de muestra seca). La determinación de minerales se hizo por espectrofotometría de absorción atómica (Termo Scientific ${ }^{\circledR}$, Modelo PAL 3000; Cambridge, Reino Unido) por medio de lámparas y curvas de calibración con estándares específicos (J. T. Baker $®)$ para cada elemento, con base en el método 965.09 de la AOAC (1990). El contenido de S y P se cuantificó con un espectrofotómetro ultravioleta-visible GBC@ (Modelo CINTRA; Melbourne, Australia). Las lecturas se hicieron por triplicado.

\section{Análisis estadístico}

Los datos de contenido de minerales en las cenizas de las poblaciones de frijol, se sometieron a análisis de varianza; se efectuaron comparaciones entre grupos de origen de poblaciones y entre poblaciones anidadas en grupos y se complementó con comparaciones múltiples de medias a través de la prueba de Tukey $(P \leq 0.05)$. Las medias se estandarizaron por el máximo valor de cada variable, y se realizó un análisis discriminante canónico con el propósito de evaluar las diferencias entre grupos de orígenes y contenido de minerales en semilla de cada población, incluyendo un análisis gráfico descriptivo de la relación entre micro y macronutrientes. Todos los análisis se realizaron en el paquete estadístico SAS (SAS Institute, 2002).

\section{RESULTADOS Y DISCUSIÓN}

El frijol común es un alimento importante en las comunidades rurales de México, no sólo por su aportación nutrimental sino por su función preventiva contra enfermedades crónico-degenerativas, incluyendo algunos tipos de cáncer (Suárez-Martínez et al. 2016). El análisis de varianza para el contenido de minerales en semilla, detectó diferencias significativas $(P \leq 0.01)$ entre grupos de 
origen de las poblaciones y entre poblaciones dentro de cada grupo; los coeficientes de variación fueron menores a 10.2 \% (Cuadro 1). La comparación de medias entre grupos de origen muestra un efecto del origen geográfico sobre el contenido de minerales en la semilla de frijol.

Worthington et al. (2012) y Soleri et al. (2013) demostraron que existen diferencias en la estructura genética de la poblaciones de frijol común conservadas por los agricultores del municipio de Santa María Jaltianguis, Oaxaca, y presuponen que entre regiones oaxaqueñas las diferencias pueden ser mayores. En este trabajo todas las poblaciones fueron sembradas en un solo sitio y con semejante manejo agronómico; en consecuencia, las diferencias entre grupos de poblaciones tienen un componente genético debido a probables diferencias en la estructura genética de las poblaciones evaluadas donde los agricultores influyeron mediante la selección de semilla.

Las poblaciones de frijol de la Sierra Norte y de los Valles Centrales mostraron frecuentemente mayores contenidos de macro y microelementos, en comparación con las originaras de la Mixteca y Sierra Sur. En los contenidos de minerales en semilla de frijol no se descarta la alta influencia ambiental debido a que solo fueron evaluadas en un sitio y una estación de cultivo. No obstante, los resultados muestran que en cada región de Oaxaca se preserva una fracción importante del acervo genético de $P$. vulgaris en México, y cada acervo regional tiene ciertos patrones diferenciales en contenido de macro y micro-elementos minerales en semilla (Cuadro 2).

En el análisis discriminante canónico se determinaron diferencias significativas entre grupos de origen de poblaciones (traza de Pillai $F=3.36$ y lambda de Wilks $F=3.52 ; P$ $\leq 0.01$ ). En la Figura 1 se observa la dispersión de las poblaciones con base en las primeras dos variables canónicas discriminantes. Los patrones de diversidad por origen geográfico presentan divergencias específicas, semejantes a los resultados de análisis de varianza y comparaciones de medias previas; las poblaciones originarias de la región Mixteca se dispersan en el cuadrante inferior izquierdo y son muy cercanas a las de Sierra Sur; las de Sierra Norte se ubican en el cuadrante inferior derecho. Las poblaciones de Valles Centrales presentaron mayor dispersión en todos los cuadrantes.

En la Figura 1 también se observa que en cada grupo de poblaciones existen muestras que sobresalen por su mayor contenido de Fe, Cu, Ca, P, S, Mn y K (cuadrante superior derecho). En este sentido, Beebe et al. (2000) encontraron patrones diferenciables en contenido de minerales en semilla de frijol dentro de cada región de origen Mesoamérica, Centroamérica y Sudamérica. En el germoplasma evaluado existen poblaciones sobresalientes con alto contenido de minerales, poblaciones factibles de utilizarse en esquemas de mejoramiento genético, tal como lo proponen Teixeira et al. (2015) en germoplasma de P. vulgaris. No obstante, es necesario evaluar si las poblaciones sobresalientes en minerales son estables a través de ambiente y además conservan altos rendimientos, considerando que las prácticas agrícolas pueden afectar su respuesta (Astudillo y Blair, 2008).

En la comparación de medias entre poblaciones (Cuadro 3), mostró que entre ellas existe alta variabilidad en contenido de macro y micro-elementos en la semilla de

Cuadro 1. Cuadrados medios y su significancia en el análisis de varianza del contenido de minerales en semilla de grupos poblacionales de frijol.

\begin{tabular}{|c|c|c|c|c|}
\hline Fuentes de variación & Grupos & Colecta/grupos & Error & CV (\%) \\
\hline & \multicolumn{4}{|c|}{ Macroelementos } \\
\hline S & $8094 * *$ & $667 \star *$ & 11.7 & 7.5 \\
\hline$P$ & $106,769 * *$ & $52,431 * *$ & 137.1 & 3.6 \\
\hline $\mathrm{Na}$ & $4327 * *$ & $1017 \star \star$ & 52.3 & 10.1 \\
\hline K & $73,606 * *$ & $26,151 * *$ & 1017.4 & 3.5 \\
\hline $\mathrm{Mg}$ & $746 * *$ & $730 * \star$ & 3.8 & 1.6 \\
\hline \multirow[t]{2}{*}{$\mathrm{Ca}$} & $998 * *$ & $1353 * *$ & 2.8 & 7.2 \\
\hline & \multicolumn{4}{|c|}{ Microelementos } \\
\hline $\mathrm{Fe}$ & $1.83 * *$ & $2.22 * \star$ & 0.1 & 6.4 \\
\hline Zn & $4.11 \star \star$ & $0.90 * *$ & 0.17 & 9.7 \\
\hline $\mathrm{Cu}$ & $6.89 * *$ & $0.97 * *$ & 0.01 & 7.4 \\
\hline $\mathrm{Mn}$ & $0.17 \star \star$ & $0.10 * *$ & 0.001 & 2.9 \\
\hline
\end{tabular}

**Significativo a P $\leq 0.01 ; \mathrm{CV}=$ coeficiente de variación. 
Cuadro 2. Comparación de medias entre grupos de poblaciones de frijol con diferentes orígenes geográficos, en contenido de minerales en semilla.

\begin{tabular}{|c|c|c|c|c|}
\hline \multirow{2}{*}{ Mineral } & \multicolumn{4}{|c|}{ Grupos (contenido en mg/100 g) } \\
\hline & Mixteca & Sierra Norte & Sierra Sur & Valles Centrales \\
\hline & \multicolumn{4}{|c|}{ Macroelementos } \\
\hline S & 39.4 c & $67.1 \mathrm{a}$ & $41.9 b$ & $40.5 \mathrm{bc}$ \\
\hline$P$ & $341.7 b$ & $266.0 \mathrm{c}$ & $267.5 c$ & $359.8 \mathrm{a}$ \\
\hline $\mathrm{Na}$ & $63.9 \mathrm{c}$ & $74.2 b$ & $70.6 b$ & $85.1 \mathrm{a}$ \\
\hline K & $918.4 b$ & $946.6 \mathrm{a}$ & $909.0 \mathrm{~b}$ & 846.40 \\
\hline $\mathrm{Mg}$ & $117.7 \mathrm{~b}$ & $118.6 b$ & $125.9 \mathrm{a}$ & 113.70 \\
\hline \multirow[t]{2}{*}{$\mathrm{Ca}$} & $91.3 d$ & $98.3 b$ & $93.6 \mathrm{c}$ & $100.1 \mathrm{a}$ \\
\hline & \multicolumn{4}{|c|}{ Microelementos } \\
\hline $\mathrm{Fe}$ & $5.24 \mathrm{a}$ & $5.11 \mathrm{a}$ & $5.11 \mathrm{a}$ & $4.87 b$ \\
\hline Zn & $4.1 \mathrm{~b}$ & $4.5 \mathrm{a}$ & $4.0 \mathrm{~b}$ & $4.7 \mathrm{a}$ \\
\hline $\mathrm{Cu}$ & $1.23 b$ & $1.14 \mathrm{c}$ & $1.25 b$ & $2.02 \mathrm{a}$ \\
\hline $\mathrm{Mn}$ & $1.24 \mathrm{~b}$ & $1.32 \mathrm{a}$ & $1.17 \mathrm{c}$ & 1.180 \\
\hline
\end{tabular}

Medias con la misma letra en cada hilera no difieren significativamente $(P \leq 0.05)$.

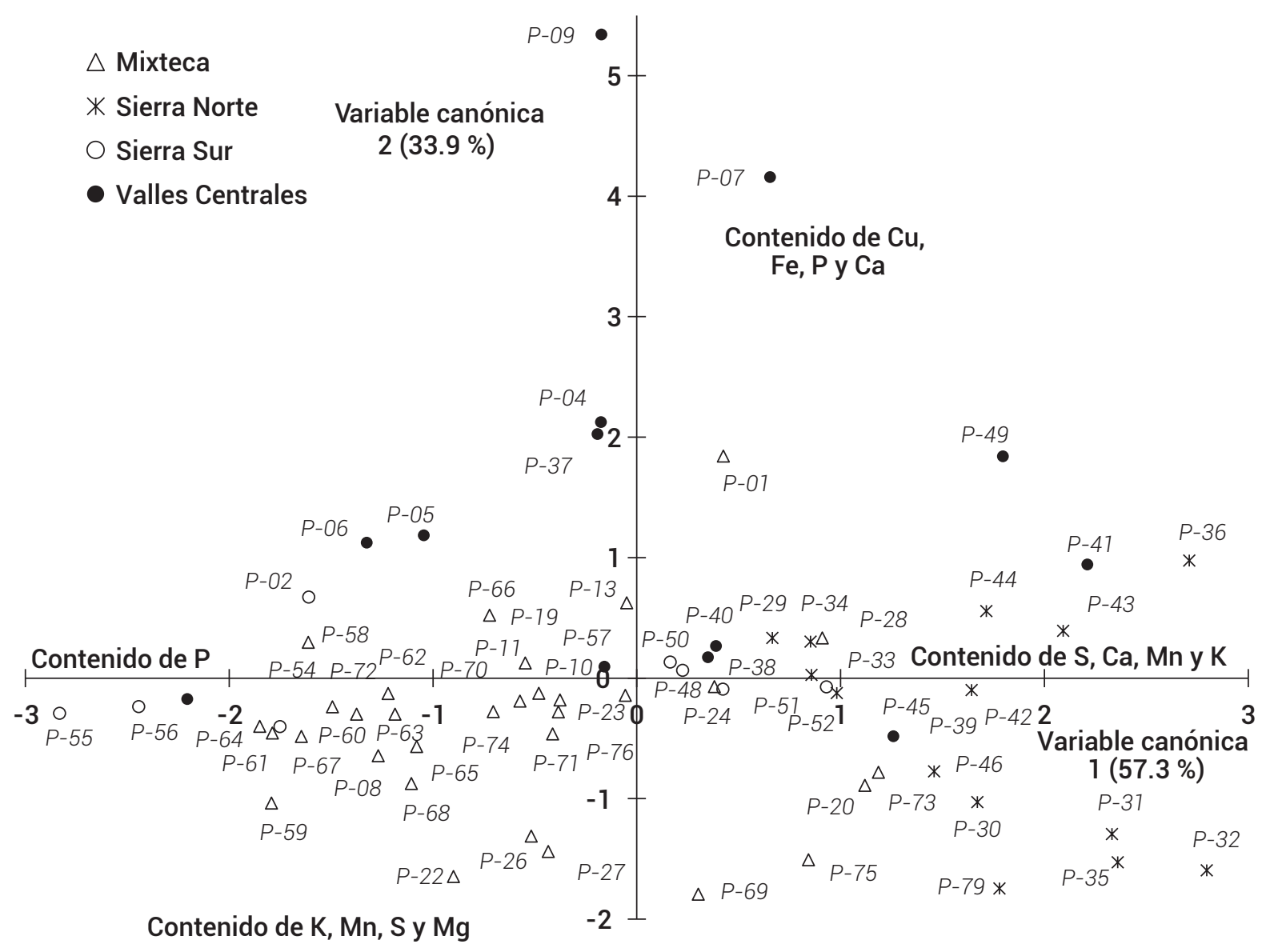

Figura 1. Dispersión de poblaciones en función de las dos primeras variables canónicas discriminantes, con base en contenido de minerales en semilla. 
frijol. En contenido de S sobresalieron dos poblaciones de la Mixteca, 11 de Sierra Norte, dos de la Sierra Sur y dos de los Valles Centrales, con valores mayores a $60 \mathrm{mg} / 100 \mathrm{~g}$ en materia seca. En relación con los valores de P, algunas poblaciones mostraron más de $490 \mathrm{mg} / 100 \mathrm{~g}$, que supera a los contenidos reportados por Martínez-Meyer et al. (2013) y Prolla et al. (2010), de 468 y 358.6 mg/100 g, respectivamente.

En contraste, en contenido de Ca los valores más altos, de 100 a $148.9 \mathrm{mg} / 100 \mathrm{~g}$ estimados en este trabajo fueron inferiores a los consignados por Prolla et al. (2010) de $399.7 \mathrm{mg} / 100 \mathrm{~g}$, y ligeramente superiores a los reportados por Martínez-Meyer et al. (2013) de 141 mg/100 g. Estas diferencias y patrones de respuesta de los materiales aquí evaluados en relación con otros reportes, señalan que existen diferencias conferidas tanto por el método de análisis como por el material genético y condiciones de cultivo, como lo demostraron Moraghan y Grafton (2001) y Prolla et al. (2010), al evaluar el efecto del genotipo, año de cosecha y condicion de almacenamiento en el contenido de minerales en frijol común.

En relación con el contenido de micro-elementos, la variación estimada de Fe y $\mathrm{Zn}$ en este trabajo se encuentra dentro del intervalo reportado por Moraghan y Grafton (2001), Prolla et al. (2010), Martínez-Meyer et al. (2013) y Gouveia et al. (2014) de 3.7 a 7.4 y de 3.3 a $6.1 \mathrm{mg} / 100 \mathrm{~g}$, respectivamente. En un estudio de más de 2000 variedades de frijol para minerales, especialmente hierro y zinc, se encontró que el contenido de hierro varió de 34 a 89 $\mathrm{mg} \mathrm{kg}^{-1}$, con un promedio de $55 \mathrm{mg} \mathrm{kg}^{-1}$ equivalente a 5.5 $\mathrm{mg} / 100 \mathrm{~g}$ (Astudillo y Blair, 2008).

Welch et al. (2000) y Teixeira et al. (2015) señalaron que un programa de mejoramiento genético de frijol, se puede iniciar utilizando como referencia el contenido de Fe y Zn; en este sentido las poblaciones sobresalientes fueron P-01 a P-04, P-06, P-07, P-46, P-60, P-61 y P-73 (Cuadro 3); es decir, dentro de cada región sobresalieron poblaciones con alto contenido de macro y micro-elementos, y se puede tener más de una opción de aprovechamiento directo, o bien iniciar programas de mejoramiento genético apoyados en análisis bioquímicos y marcadores moleculares para incrementar la eficiencia en la selección.

La Figura 2 presenta la dispersión de poblaciones evaluadas en función del contenido total de macro y microelementos. La división en cuatro cuadrantes en función del promedio de macro y micro-nutrientes generó cuatro escenarios para las poblaciones; en el cuadrante superior izquierdo se ubican las poblaciones con mayor contenido de micro-elementos pero bajo en macro-elementos; en contraste, en el cuadrante inferior derecho, están las de alto valor en macro y bajo en micro-elementos.

Las poblaciones sobresalientes, por arriba de las medias, tanto en macro como en micro-elementos se localizan en el cuadrante superior derecho, donde se ubicaron seis poblaciones de la Sierra Norte, cuatro de los Valles Centrales y nueve de la Mixteca, las que representan 42.9, 30.8 y 29.0 \% del total de poblaciones evaluadas de cada región, respectivamente. Específicamente, la colecta P-06 destacó por su alto contenido de micro y macro-elementos, en tanto que las colectas P-60, P-67 y P-75 de la Mixteca y la P-79 de Sierra Norte, contienen mayor cantidad de macro-elementos y un poco más que el promedio general de micro-elementos.

\section{CONCLUSIONES}

Los análisis del contenido de minerales en la semilla de poblaciones nativas de frijol común de diferente origen geográfico del estado de Oaxaca, mostraron diferencias entre y dentro de grupos. Las poblaciones de los Valles Centrales fueron superiores en contenidos de $\mathrm{P}, \mathrm{Na}, \mathrm{Ca}, \mathrm{Zn}$ y Cu, y el grupo de la Sierra Norte sobresalió en S, K, Fe, Zn y $\mathrm{Mn}$. Las evaluaciones de micro y macro-elementos minerales totales permitieron identificar poblaciones de alto contenido, como las colectas P-06, P-60, P-67, P-73, P-75 y P-79. En contenido de Fe y Zn, cuyas deficiencias están asociadas con mala nutrición y problemas de salud, las poblaciones sobresalientes fueron P-01, P-03, P-04, P-06 y P-07.

\section{BIBLIOGRAFÍA}

AOAC-Association of Official Analytical Chemists (1990) Official Methods of Analysis of the Association of Official Analytical Chemists. Vol. 2, 15th ed., Association of Official Analytical Chemist, Washington DC, USA. pp:27-42.

Astudillo C. y M. W. Blair (2008) Contenido de hierro y zinc en la semilla y su respuesta al nivel de fertilización con fósforo en 40 variedades de frijol colombianas. Agronomía Colombiana 26:471-476.

Bazzano L. A., J. He, L. G. Ogden, C. Loria, S. Vupputuri, L. Myers and P. K. Whelton (2001) Legume consumption and risk of coronary heart disease in US men and women. Archives of Internal Medicine 161:2573-2578.

Beebe S., A. Gonzalez and J. Rengifo (2000) Research on trace minerals in the common bean. Food and Nutrition Bulletin 21:387-391.

Bidoli E., R. Talamini, C. Bosetti, E. Negri, D. Maruzzi, M. Montella, S. Franceschi and C. La Vecchia (2005) Macronutrients, fatty acids, cholesterol and prostate cancer risk. Annals of Oncology 16:152-157.

Bitocchi E., L. Nanni, E. Bellucci, M. Rossi, A. Giardini, P. S. Zeuli, G. Logozzo, J. Stougaard, P. McClean, G. Attene and R. Papa (2012) Mesoamerican origin of the common bean (Phaseolus vulgaris L.) is revealed by sequence data. Proceedings of the National Academy of Sciences of the USA 109:E788-E796.

Espinosa-Alonso L.G., A. Lygin, J. M. Widholm, M. E. Valverde and O. ParedesLópez (2006) Polyphenols in wild and weedy Mexican common beans (Phaseolus vulgaris L.). Journal of Agricultural and Food Chemistry 54:4436-4444

FAOSTAT (2015) Anuario Estadístico 2014. Dirección de Estadística, Organización de las Naciones Unidas para la Alimentación y la Agricultura. Roma, Italia. http://faostat3.fao.org/download/Q/ 
Cuadro 3. Contenido de minerales $(\mathrm{mg} / 100 \mathrm{~g})$ en semilla de frijol originario de cuatro regiones del estado de Oaxaca.

\begin{tabular}{|c|c|c|c|c|c|c|c|c|c|c|}
\hline \multirow{2}{*}{ Población } & \multicolumn{6}{|c|}{ Macroelementos } & \multicolumn{4}{|c|}{ Microelementos } \\
\hline & $S$ & $P$ & K & $\mathrm{Mg}$ & $\mathrm{Ca}$ & $\mathrm{Na}$ & Zn & $\mathrm{Mn}$ & $\mathrm{Cu}$ & $\mathrm{Fe}$ \\
\hline \multicolumn{11}{|c|}{ Grupo de poblaciones de La Mixteca } \\
\hline P-01 & $27.0^{+}$ & 432.5 & 847.6 & 113.2 & 123.5 & 57.0 & 5.0 & 1.3 & 2.4 & 7.4 \\
\hline P-08 & 27.5 & 318.2 & 907.0 & 119.3 & 102.5 & 46.8 & 4.1 & 1.3 & 1.1 & 5.1 \\
\hline P-10 & 29.1 & 423.5 & 889.9 & 102.7 & 81.3 & 59.1 & 5.2 & 1.2 & 1.6 & 5.2 \\
\hline P-11 & 41.4 & 359.4 & 969.6 & 122.3 & 105.6 & 50.5 & 4.4 & 1.2 & 1.2 & 4.3 \\
\hline P-13 & 37.5 & 369.7 & 755.6 & 92.2 & 83.4 & 101.6 & 3.8 & 1.0 & 1.3 & 3.9 \\
\hline P-19 & 34.6 & 330.8 & 810.8 & 98.5 & 87.5 & 41.0 & 4.2 & 1.2 & 1.1 & 3.8 \\
\hline P-20 & 59.7 & 313.7 & 1022.6 & 124.3 & 111.1 & 71.5 & 4.6 & 1.4 & 0.9 & 4.9 \\
\hline P-22 & 25.2 & 404.1 & 931.9 & 101.1 & 77.2 & 42.8 & 4.9 & 1.3 & 0.9 & 4.6 \\
\hline P-23 & 40.5 & 405.2 & 911.9 & 97.7 & 73.7 & 74.4 & 4.3 & 1.0 & 1.2 & 3.7 \\
\hline P-24 & 33.1 & 421.2 & 815.4 & 112.1 & 145.7 & 52.2 & 4.6 & 1.7 & 1.2 & 5.7 \\
\hline P-26 & 42.4 & 359.4 & 1012.8 & 119.4 & 93.4 & 64.8 & 4.7 & 1.3 & 0.8 & 4.2 \\
\hline P-27 & 24.2 & 415.5 & 942.7 & 111.8 & 99.5 & 92.0 & 4.9 & 1.7 & 1.5 & 5.6 \\
\hline P-28 & 54.9 & 392.6 & 754.2 & 97.1 & 87.2 & 107.5 & 4.6 & 1.1 & 1.2 & 4.0 \\
\hline P-58 & 26.2 & 298.8 & 846.9 & 105.2 & 69.5 & 74.5 & 3.9 & 0.9 & 1.3 & 4.3 \\
\hline P-59 & 26.6 & 290.8 & 957.9 & 115.5 & 58.8 & 40.7 & 3.5 & 1.1 & 1.3 & 4.9 \\
\hline P-60 & 35.5 & 581.5 & 1013.5 & 148.3 & 143.0 & 62.0 & 3.9 & 1.5 & 1.2 & 6.4 \\
\hline P-61 & 33.0 & 196.9 & 886.0 & 138.3 & 106.2 & 74.1 & 3.8 & 1.4 & 1.2 & 6.0 \\
\hline P-62 & 34.6 & 109.9 & 854.7 & 114.8 & 75.0 & 72.1 & 3.8 & 1.1 & 1.2 & 4.3 \\
\hline P-63 & 32.6 & 318.2 & 923.8 & 104.4 & 60.9 & 58.5 & 3.8 & 0.9 & 1.2 & 3.7 \\
\hline P-64 & 34.5 & 675.3 & 918.7 & 122.2 & 96.9 & 62.0 & 3.6 & 1.2 & 1.2 & 3.9 \\
\hline P-65 & 43.4 & 196.9 & 990.2 & 135.8 & 96.3 & 51.6 & 3.7 & 1.4 & 1.2 & 4.6 \\
\hline P-66 & 34.6 & 89.3 & 882.6 & 107.9 & 76.6 & 72.0 & 4.1 & 1.0 & 1.4 & 3.9 \\
\hline P-67 & 41.4 & 666.1 & 977.1 & 138.4 & 96.9 & 65.3 & 4.2 & 1.2 & 1.4 & 5.6 \\
\hline P-68 & 45.4 & 249.5 & 887.9 & 137.5 & 95.2 & 63.3 & 3.6 & 1.3 & 1.1 & 6.1 \\
\hline P-69 & 48.9 & 99.5 & 1042.5 & 111.2 & 53.8 & 72.5 & 3.8 & 1.2 & 0.9 & 4.5 \\
\hline P-70 & 43.5 & 330.8 & 913.6 & 118.9 & 81.0 & 66.1 & 3.8 & 1.1 & 1.3 & 4.6 \\
\hline P-71 & 42.5 & 346.8 & 807.9 & 107.2 & 80.3 & 56.3 & 3.7 & 1.1 & 1.1 & 4.7 \\
\hline P-72 & 49.3 & 492.2 & 881.9 & 133.2 & 95.3 & 78.0 & 3.3 & 1.2 & 1.1 & 4.1 \\
\hline P-73 & 62.2 & 183.1 & 1214.7 & 147.8 & 109.1 & 70.2 & 4.3 & 1.4 & 1.4 & 6.7 \\
\hline P-74 & 40.5 & 149.9 & 833.9 & 114.3 & 90.2 & 68.2 & 3.7 & 1.2 & 1.0 & 4.7 \\
\hline P-75 & 67.2 & 542.6 & 1159.1 & 143.0 & 97.0 & 55.0 & 3.6 & 1.5 & 1.3 & 5.9 \\
\hline P-76 & 43.5 & 121.3 & 825.0 & 110.3 & 72.9 & 49.9 & 3.8 & 1.1 & 1.2 & 4.5 \\
\hline \multicolumn{11}{|c|}{ Grupo de poblaciones de Sierra Norte } \\
\hline P-29 & 45.5 & 327.4 & 846.7 & 93.3 & 79.7 & 66.9 & 4.5 & 1.0 & 1.3 & 3.8 \\
\hline P-30 & 60.8 & 325.1 & 906.1 & 107.3 & 80.2 & 77.2 & 4.6 & 1.3 & 1.2 & 5.4 \\
\hline P-31 & 72.1 & 141.9 & 1060.9 & 128.6 & 125.4 & 75.3 & 4.5 & 1.6 & 0.6 & 5.0 \\
\hline P-32 & 81.0 & 231.2 & 1089.7 & 127.6 & 100.9 & 62.1 & 4.9 & 1.6 & 0.9 & 5.4 \\
\hline
\end{tabular}


Cuadro 3. Continuación.

\begin{tabular}{|c|c|c|c|c|c|c|c|c|c|c|}
\hline \multirow{2}{*}{ Población } & \multicolumn{6}{|c|}{ Macroelementos } & \multicolumn{4}{|c|}{ Microelementos } \\
\hline & $S$ & $\mathrm{P}$ & $K$ & $\mathrm{Mg}$ & $\mathrm{Ca}$ & $\mathrm{Na}$ & $\mathrm{Zn}$ & $\mathrm{Mn}$ & $\mathrm{Cu}$ & $\mathrm{Fe}$ \\
\hline P-33 & 51.4 & 302.2 & 811.9 & 89.9 & 61.3 & 96.6 & 4.7 & 0.9 & 1.2 & 3.9 \\
\hline P-34 & 47.4 & 283.9 & 821.5 & 95.8 & 87.7 & 70.4 & 4.3 & 1.1 & 1.1 & 4.4 \\
\hline P-35 & 74.1 & 107.6 & 985.4 & 123.2 & 112.0 & 62.0 & 4.4 & 1.6 & 0.6 & 5.0 \\
\hline P-36 & 76.1 & 219.7 & 965.5 & 119.0 & 113.4 & 84.2 & 4.7 & 1.3 & 1.5 & 4.7 \\
\hline P-42 & 60.3 & 265.6 & 929.8 & 112.7 & 86.5 & 76.7 & 4.9 & 1.3 & 1.5 & 5.5 \\
\hline$P-43$ & 61.8 & 286.2 & 829.1 & 102.8 & 97.7 & 81.4 & 4.0 & 1.1 & 1.3 & 5.6 \\
\hline$P-44$ & 74.1 & 201.5 & 905.4 & 132.6 & 118.0 & 82.6 & 3.7 & 1.3 & 1.3 & 5.6 \\
\hline$P-45$ & 80.0 & 261.0 & 1001.4 & 151.3 & 120.9 & 74.9 & 4.8 & 1.3 & 0.9 & 5.7 \\
\hline P-46 & 80.0 & 222.1 & 1028.5 & 146.9 & 112.7 & 73.6 & 4.6 & 1.3 & 0.9 & 6.4 \\
\hline P-79 & 75.1 & 549.3 & 1070.1 & 129.8 & 79.9 & 67.8 & 3.7 & 1.6 & 1.6 & 5.2 \\
\hline \multicolumn{11}{|c|}{ Grupo de poblaciones de Sierra Sur } \\
\hline P-02 & $16.6^{+}$ & 336.4 & 843.9 & 121.1 & 132.3 & 68.5 & 5.2 & 1.5 & 1.5 & 5.2 \\
\hline P-48 & 72.1 & 169.4 & 986.7 & 144.6 & 93.4 & 76.1 & 3.6 & 1.2 & 1.2 & 4.8 \\
\hline P-50 & 56.4 & 307.9 & 862.9 & 118.2 & 84.0 & 71.1 & 3.6 & 1.1 & 1.2 & 4.8 \\
\hline P-51 & 54.9 & 322.8 & 853.4 & 109.9 & 69.6 & 67.0 & 4.0 & 0.9 & 1.3 & 5.4 \\
\hline P-52 & 67.1 & 233.5 & 936.3 & 132.0 & 99.4 & 71.0 & 4.3 & 1.2 & 1.2 & 5.7 \\
\hline P-54 & 26.2 & 290.7 & 872.3 & 112.0 & 76.9 & 70.9 & 3.8 & 1.1 & 1.2 & 4.5 \\
\hline P-55 & 19.6 & 248.4 & 982.8 & 133.0 & 85.9 & 74.5 & 3.9 & 1.1 & 1.3 & 4.9 \\
\hline P-56 & 22.6 & 224.3 & 933.8 & 136.3 & 107.2 & 69.9 & 3.8 & 1.2 & 1.1 & 5.6 \\
\hline \multicolumn{11}{|c|}{ Grupo de poblaciones de Valles Centrales } \\
\hline P-03 & 22.8 & 440.9 & 784.2 & 111.3 & 117.8 & 114.4 & 6.1 & 1.3 & 2.5 & 6.9 \\
\hline P-04 & 13.3 & 496.7 & 783.0 & 101.4 & 85.3 & 150.1 & 5.4 & 1.2 & 2.5 & 6.1 \\
\hline P-05 & 20.2 & 465.9 & 799.8 & 106.1 & 92.5 & 100.9 & 5.7 & 1.1 & 2.0 & 5.0 \\
\hline P-06 & 27.1 & 778.3 & 845.0 & 103.8 & 72.7 & 73.9 & 5.2 & 1.2 & 4.6 & 6.7 \\
\hline P-07 & 25.8 & 413.6 & 811.7 & 125.1 & 116.1 & 67.7 & 5.2 & 1.2 & 4.2 & 6.6 \\
\hline P-09 & 31.5 & 327.4 & 781.5 & 118.8 & 148.9 & 82.6 & 4.7 & 1.5 & 1.8 & 5.0 \\
\hline P-37 & 52.9 & 310.2 & 825.9 & 105.2 & 81.6 & 79.3 & 4.1 & 1.0 & 1.1 & 4.1 \\
\hline P-38 & 55.9 & 283.9 & 842.3 & 103.9 & 86.4 & 84.6 & 4.2 & 1.2 & 1.1 & 5.1 \\
\hline P-39 & 48.4 & 301.0 & 841.6 & 98.1 & 75.5 & 63.1 & 4.3 & 1.0 & 1.2 & 3.7 \\
\hline$P-40$ & 70.1 & 228.9 & 857.6 & 116.9 & 117.7 & 68.1 & 4.9 & 1.3 & 1.4 & 5.2 \\
\hline P-41 & 76.1 & 196.9 & 880.8 & 132.3 & 133.1 & 66.6 & 3.7 & 1.3 & 1.4 & 4.7 \\
\hline P-49 & 54.4 & 326.2 & 858.8 & 113.9 & 73.8 & 73.6 & 3.8 & 0.9 & 1.1 & 4.4 \\
\hline$P-57$ & 23.6 & 247.2 & 1090.5 & 141.1 & 111.3 & 74.1 & 3.6 & 1.2 & 1.2 & 5.4 \\
\hline DSH-Tukey ${ }^{\dagger+}$ & 14.9 & 51.0 & 139.0 & 8.5 & 7.3 & 31.5 & 1.8 & 0.1 & 0.4 & 1.4 \\
\hline
\end{tabular}




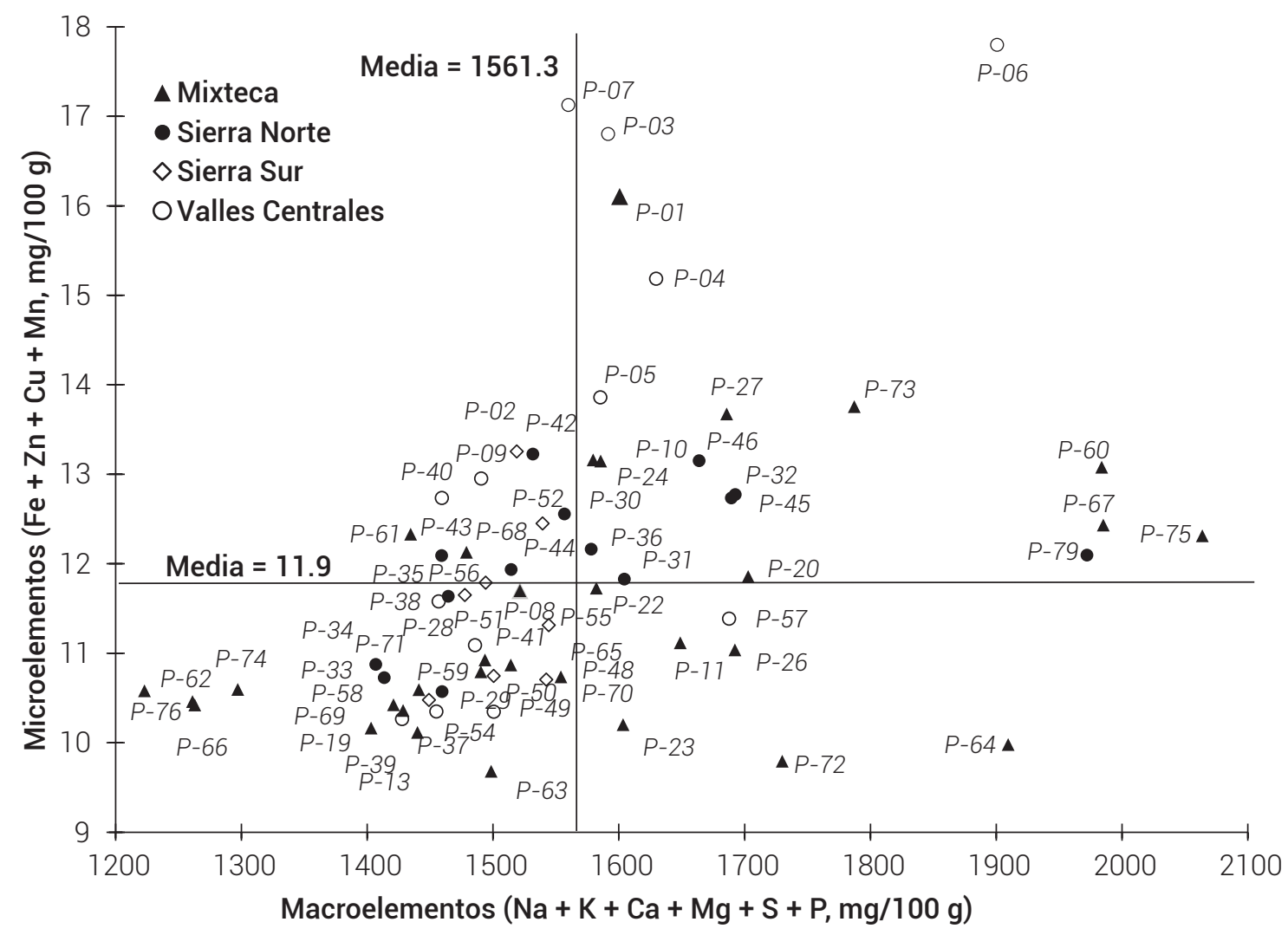

Figura 2. Dispersión de poblaciones evaluadas en relación con el contenido total de macro y micro-elementos minerales en semilla de poblaciones de frijol común.

QC/S (Octubre 2015).

Gouveia C. S. S., G. Freitas, J. H. de Brito, J. J. Slaski and M. A. A. Pinheiro de Carbalho (2014) Nutritional and mineral variability in 52 accessions of common bean varieties (Phaseolus vulgaris L.) from Madeira Island. Agricultural Sciences 5:317-329.

Guzmán M. S. H., J. A. Acosta G., M. A. Álvarez-Muñoz, S. García-Delgado y G. Loarca-Piña (2002) Calidad alimentaria y potencial nutracéutico del frijol (Phaseolus vulgaris L.). Agricultura Técnica en México 28:159-173

Hernández-López V. M., M. L. P. Vargas-Vázquez, J. S. Muruaga-Martínez, S. Hernández-Delgado y N. Mayek-Pérez (2013) Origen, domesticación y diversificación del frijol común. Avances y perspectivas. Revista Fitotecnia Mexicana 36:95-104.

Kabagambe E. K., A. Baylin, E. Ruiz-Narvarez, X. Siles and H. Campos (2005) Decreased consumption of dried mature beans is positively associated with urbanization and nonfatal acute myocardial infarction. Journal of Nutrition 135:1770-1775.

Lépiz I. R., J. J. López A., J. J. Sánchez G., F. Santacruz-Ruvalcaba, R. Nuño R. y E. Rodríguez G. (2010) Características morfológicas de formas cultivadas, silvestres e intermedias de frijol común de hábito trepador. Revista Fitotecnia Mexicana 33:21-28.

Martínez-Meyer M. R., A. Rojas, A. Santanen and F. L. Stoddard (2013) Content of zinc, iron and their absorption inhibitors in Nicaraguan common beans (Phaseolus vulgaris L.). Food Chemistry 136:87-93

Moraghan J. T. and K. Grafton (2001) Genetic diversity and mineral composition of common bean seed. Journal of the Science of Food and Agriculture 81:404-408.

Muñoz-Velázquez E. E., D. Rubio-Hernández, I. Bernal-Lugo, R. Garza-García y C. Jacinto-Hernández (2009) Caracterización de genotipos nativos de frijol del estado de Hidalgo, con base a calidad del grano. Agricultura Técnica en México 35:426-435.
Prolla I. R. D., R. G. Barbosa, A. P. L. Veeck, P. R. August, L. P. da Silva, N. D. Ribeiro and T. Emanuelli (2010) Cultivar, harvest year and storage conditions affecting nutritional quality of common beans (Phaseolus vulgaris L.). Ciência e Tecnologia de Alimentos 30 (Supl. 1):96-102.

Raya-Pérez J. C., G. M. Gutiérrez-Benicio, J. G. Ramírez P., J. CovarrubiasPrieto y C. L. Aguirre-Mancilla (2014) Caracterización de proteínas y contenido mineral de dos variedades nativas de frijol de México. Agronomía Mesoamericana 25:01-11.

Reynoso C. R., M. C. Ríos U., I. Torres P., J. A. Acosta G., A. C. Palomino S., M. Ramos G., E. González J. y S. H. Guzmán M. (2007) El consumo de frijol común (Phaseolus vulgaris L.) y su efecto sobre cáncer de colon en ratas Spraque-Dawley. Agricultura Técnica en México 33:43-52

Salinas-Moreno Y., L. Rojas-Herrera, E. Sosa-Montes y P. Pérez-Herrera (2005) Composición de antocianinas en variedades de frijol negro (Phaseolus vulgaris L.) cultivadas en México. Agrociencia 39:385-394.

SAS Institute (2002) SAS software release version 9.0. Statistical Analysis System for Windows. SAS Institute Inc. Cary, NC. USA.

SIAP-Servicio de Información Agroalimentaria y Pesquera (2014) Anuario estadístico de la producción agrícola 2014. Servicio de Información Agroalimentaria y Pesquera, Secretaría de Agricultura, Desarrollo Rural, Pesca y Alimentación. México, D. F. http:// www.siap.gob.mx/cierre-de-la-produccion-agricola-por-cultivo/ (Octubre 2015).

Singh S. P., P. Gepts and D. G. Debouck (1991) Races of common bean (Phaseolus vulgaris, Fabaceae). Economic Botany 45:379-396.

Soleri D., M. Worthington, F. Aragón-Cuevas, S. E. Smith and P. Gepts (2013) Farmers' varietal identification in a reference sample of local Phaseolus species in the Sierra Juárez, Oaxaca, Mexico. Economic Botany 67:283-298. 
Suárez-Martínez S. E., R. A. Ferriz-Martínez, R. Campos-Vega, J. E. Elton-Puente, K. de la Torre-Carbot and T. García-Gasca (2016) Bean seeds: leading nutraceutical source for human health. CyTA-Journal of Food 14:131-137.

Teixeira R. K. S., D. C. Lima, A. F. B. Abreu and M. A. P. Ramalho (2015) Implications of early selection for grain colour on iron and zinc content and productivity of common bean. Plant Breeding 134:193-196.

Vargas-Vázquez M. L. P., J. S. Muruaga-Martínez, P. Pérez-Herrera, H. R. GillLangarica, G. Esquivel-Esquivel, M. A. Martínez-Damián, R. RosalesSerna y N. Mayek-Pérez (2008) Caracterización morfoagronómica de la colección núcleo de la forma cultivada de frijol común del INIFAP. Agrociencia 42:787-797.

Vidal-Barahona A., L. C. Lagunes-Espinoza, E. Valadez M. y C. F. Ortiz-García (2006) Variabilidad morfológica y molecular de cultivares crio-
Ilos y mejorados de frijol común en Tabasco, México. Revista Fitotecnia Mexicana 29:273-281.

Welch R. M. (2008) Linkages between trace elements in food crops and human health. In: Micronutrient Deficiencies in Global Crop Production. Alloway B. J. (ed.). Springer Netherlands, Heidelberg, Germany. pp:287-309.

Welch R. M., W. A. House, S. Beebe and Z. Cheng (2000) Genetic selection for enhanced bioavailable levels of iron in bean (Phaseolus vulgaris L.) seeds. Journal of Agricultural and Food Chemistry 48:3576-3580.

Worthington M., D. Soleri, F. Aragón-Cuevas and P. Gepts (2012) Genetic composition and spatial distribution of farmer-managed Phaseolus bean planting: an example from a village in Oaxaca Mexico. Crop Science 52:1721-1735. 